\title{
In-situ Topographical and Elemental Characterisation of Biological Soft Matter
}

\author{
C.L. Collins ${ }^{1}$, C. McCarthy ${ }^{1}$, S.R. Burgess ${ }^{1}$ \\ ${ }^{1}$ Oxford Instruments Nanoanalysis, Halifax Road, High Wycombe, HP12 3SE, UK
}

Since the $17^{\text {th }}$ century, light microscopy (LM) has been used to gain topographical and structural information about soft biological matter, however, it has limited spatial resolution and gives no information about the chemical composition of the structures. Fluorescence light microscopy (FLM) gives more detail about the antibody or protein responses of live cells but is localised to a specific chemical reaction. As a technique, it is offers some information about the organic matrix, but no wider chemical information about surrounding structures or the inorganic composition of the sample.

Electron microscopy (EM) combined with Energy Dispersive Spectroscopy (EDS) offers biological analysts a way of simultaneously characterising their samples both structurally and chemically and at a much higher spatial resolution than can be achieved in either LM or FLM - limited only by the performance of the microscope. There is also no need to add any chemical markers or fluorophores.

The introduction of large area EDS detectors has changed soft matter biological analysis. Large active area detectors $\left(50-150 \mathrm{~mm}^{2}\right)$ with excellent light element performance and greatly improved collection efficiencies [1,2] mean increased counts and improved prospects for elemental analysis in the EM. The increased speed of collection also opens the possibility for large scale elemental characterisation. This is important in biological analysis where samples are often inhomogeneous.

Here, we present application examples of soft matter biological tissue analysed with Oxford Instruments $\mathrm{X}-\mathrm{Max}^{\mathrm{N}} 150 \mathrm{~mm}^{2}$ SDD detectors.

Figure 1 shows a human tissue retrieved from surgical biopsy from a patient who has had a metal-onmetal total hip replacement. Such prosthetics are known to physically degrade with use [3]. Wear nanoparticles are often found in the surrounding tissue, but until recently, there was no easy way to identify and characterise the different fragments. This tissue sample was prepared by embedding in a resin then sectioned into semithin sections $(\sim 1 \mu \mathrm{m}$ thick) which were then laid onto a pure silicon wafer for analysis in the SEM.

The sample was analysed using a Tescan Mira FEGSEM fitted with a multiple EDS detector system offering a combined active area of $600 \mathrm{~mm}^{2}$. Data was collected at $10 \mathrm{kV}$ with an input count rate of 60kcps. The total collection time was 15 minutes. The results clearly show the chemical composition of the steel fragment as it lies embedded in the tissue.

Figure 2 shows the importance of large scale characterisation. The sample is a whole wheat plant stem which was embedded in resin and planed down to the area of interest. A number of individual $\mathrm{X}$-ray maps (66 in total) were collected and montaged together to create a single image $(2.8 \mathrm{~mm}$ in width) composed entirely of X-ray elemental information - there is no electron image included in this data. This removes topographical contrast. The widely varying distributions of the elements only become clear at low magnifications. The $\mathrm{Cl}$ and $\mathrm{Os}$ are artefacts from the sample preparation method used but offer structural information while the K and $\mathrm{S}$ are naturally present. 


\section{Conclusions:}

The introduction of new large area SDD EDS detectors offers a new way of collecting important insitu information about biological soft matter and tissues. Fast mapping allows large areas of sample to be analysed and viewed as a whole. The result is that truly informative chemical information data can now be collected on beam sensitive materials without compromising the sample or the data acquired.
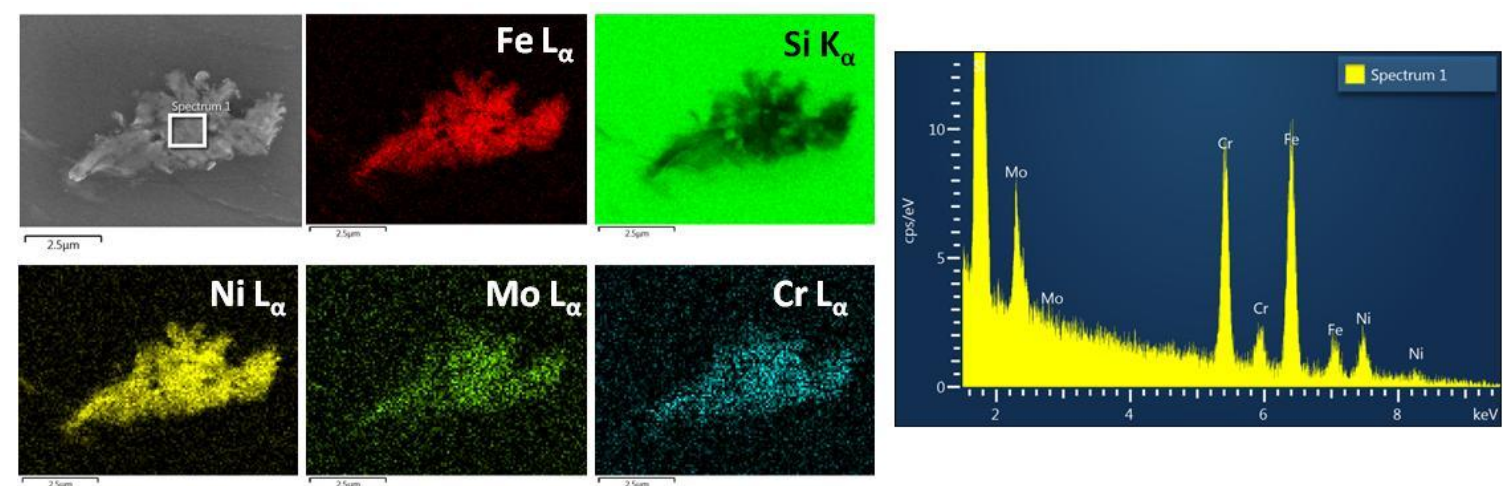

Figure 1. Human tissue biopsy from a metal hip replacement semi-thin section $(1 \mu \mathrm{m}$ thick $)$ on silicon wafer. Maps were acquired at $10 \mathrm{kV}$ with an input count rate of $60 \mathrm{kcps}$.
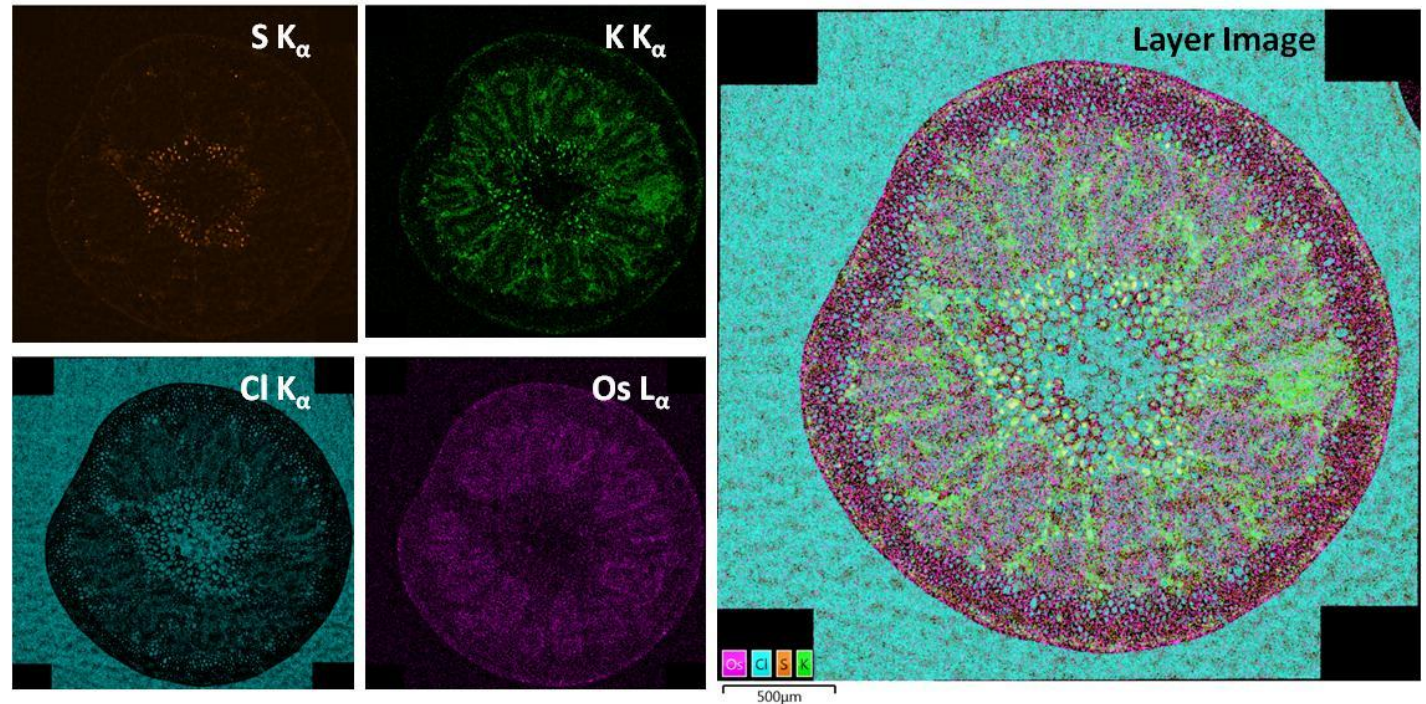

Figure 2 Large area X-ray map cross section of a whole stem of a wheat plant composed of 66 individual $x$-ray maps collected at $25 \mathrm{kV}$ and montaged together to create a single image. Image width $=2.8 \mathrm{~mm}$.

\section{References:}

[1] S Burgess et al, Microscopy \& Microanalysis (2011), p 1176-1177

[2] A Hyde et al, Microscopy \& Microanalysis (2013), p 1312-1313

[3] J. Jacobs et al, J.Bone Joint Surg Am. 80 (10) (1998) p 1447-58.

[4] Acknowledgements: Oxford Instruments are very grateful to Dr. Zhidao Xia from the Centre for Nanohealth, Swansea and Dr. Giorgio Perino from the Hospital for Special Surgery, New York for providing the tissue biopsy sample shown in Figure 1 and Jean Devonshire of Rothamsted Research for the wheat sample shown in Figure 2. 